\title{
AQUA (Advanced Query User Interface Architecture)
}

\author{
László Kovács, András Micsik, Balázs Pataki, István Zsámboki \\ MTA SZTAKI \\ Computer and Automation Research Institute \\ of the Hungarian Academy of Sciences \\ Department of Distributed Systems \\ H-1111 Budapest XI. Lágymányosi u. 11. Hungary \\ \{laszlo.kovacs,micsik,pataki,zsamboki\}@sztaki.hu
}

\begin{abstract}
AQUA is an experimental query interface which supports iterative query refinement. Currently, it can be used as an alternative query interface for NCSTRL (Networked Computer Science Technical Reports Library) and ETRDL (ERCIM Technical Reference Digital Library). As a demonstration for the extensibility of the AQUA user interface paradigm, rating facility has been added to the system.
\end{abstract}

\section{Introduction to AQUA}

AQUA is a prototype implementation of a general user interface paradigm, which is capable of modelling iterative query refinement and embedding several common and advanced query techniques. The objective of this interface is not only to provide an integrated view for different searching and browsing techniques, but also to help the user to explore the contents of a digital library.

AQUA uses a single list of panels to visualize the user's query refinement steps. The first panel applies the first query operation on the whole document collection, then each panel represents an operation on the results of the previous panel. In this way users can build complex queries step by step.

AQUA currently works with the data collections of NCSTRL (Networked Computer Science Technical Reports Library) [9] and ETRDL (ERCIM Technical Reference Digital Library) [2,4]. The screenshot on Fig. 1 shows an example query: the lower part of the AQUA screen contains a scrollable list of panels. In the example the first panel contains a boolean search for the term "digital library", with 183 results from two different digital library servers. The second panel refines the first query by searching for terms "Dienst" or "NCSTRL", and the remaining 23 documents are browsed by publication year in the third panel. The fourth panel shows the authors of documents for the years selected in the third panel. The upper part of the AQUA window serves as a console where the user can follow what is happening within the system and how their inputs are being processed. Between the console and the panel list there are tools to manipulate the whole panel list, for example queries can be saved and later reapplied to the collection. 


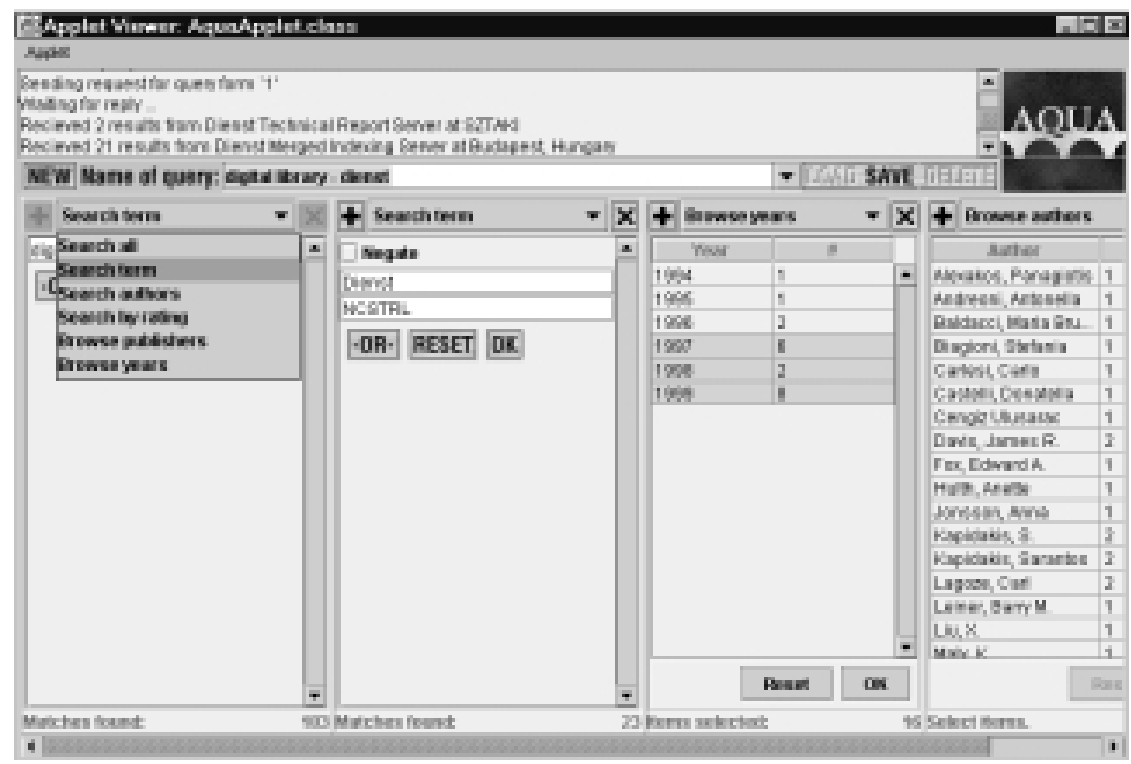

Fig. 1. A query session using AQUA

Panels in AQUA contain some standard elements: a drop-down select box at the top of the panel to change the type of query. The "delete" button in the upper right corner can be used to remove the panel from the list. The "add" button in the upper left corner is for inserting a new panel into the list before the current panel.

Both the AQUA client and the server has been implemented in Java. The client can be used as an applet, it displays the actual state of the query (e.g. Fig. 1) computed by the AQUA server. The AQUA server handles multiple client sessions, and it can access several document collections in parallel. Wrappers are used to connect different digital library systems with the server.

AQUA is available for the public since 1998 [3]. The first version was followed by a Java Swing based version in 1999. AQUA-2 has an improved user interface and provides additional features for collaborative filtering (detailed in the next section). A more detailed description of AQUA together with relations to other projects and results can be found in [5].

\section{Rating documents with AQUA}

AQUA is further developed towards a collaborative tool for digital library community use. AQUA-2 helps opinion sharing and information filtering within digital document collections. This is achieved by adding rating as a new facility to AQUA-2. Users may rate documents in the collection, and this rating information can be used as other usual document metadata during queries in AQUA. 
This work has been done in collaboration with the SELECT (EC Telematics Applications Programme) project [1]. The aim of the SELECT project is to provide methods for Internet users to filter the large amount of available information using collaborative techniques.

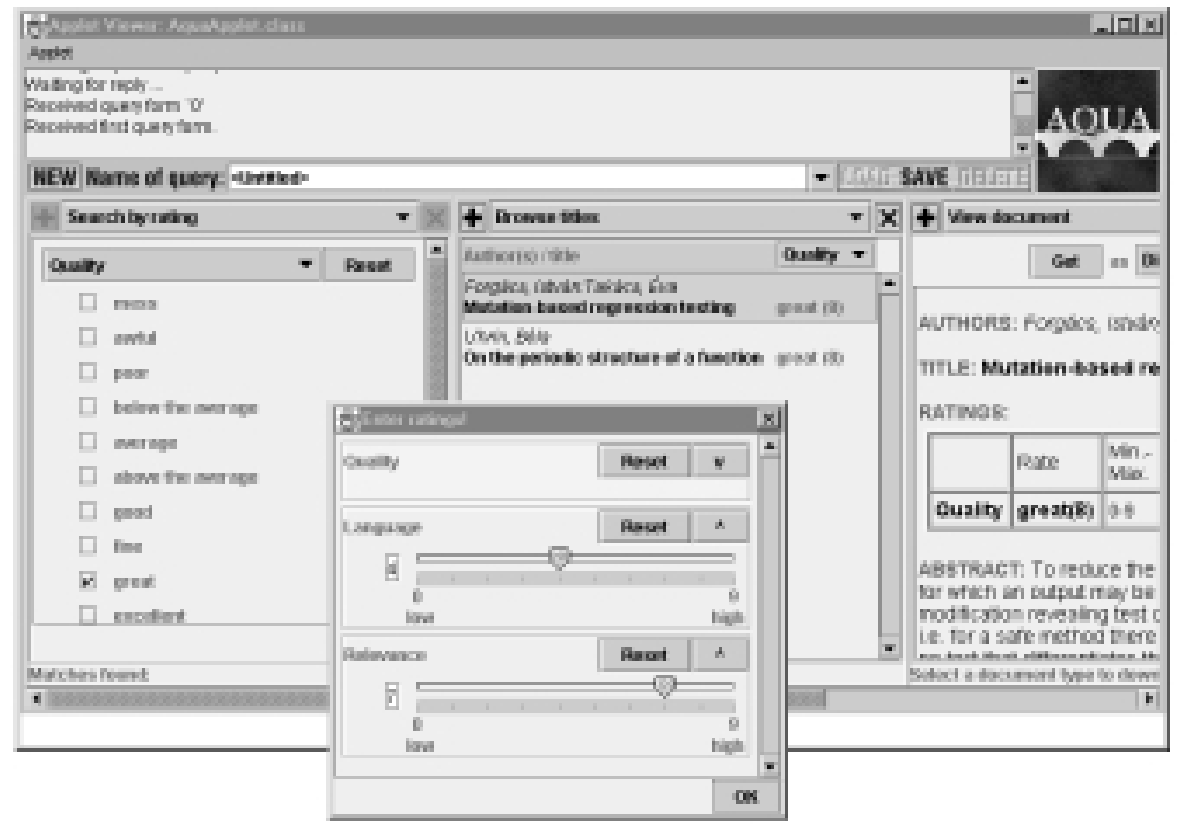

Fig. 2. Rating in AQUA

The concepts for user rating is similar to the concepts of SELECT. Users may rate each document in several categories. Categories can be defined dynamically with a given numeric scale or a set of labels. Icons and label translations for several languages can also be added to categories. User rates may have a given context. Within a context, users or user groups may have a competence value, which represents the users' expertise. Aggregate rating values for documents are calculated from rates in a single category, and presented to users. The calculation of these aggregate values may rely on competence levels, providing an architecture for many variations of rating (e.g. peer reviews, public opinions).

The user interface has been augmented with a rating input dialog, various rating displays and a new panel (Fig. 2). Aggregate rating values are shown in the List titles and Document panels. Document panels also show users' own rates for documents, and a new button activates the dialog for adding or changing rating values. A completely new panel type (Search by rating) was introduced to filter documents according to their rating values. This means that a result set can be filtered by rating values at any point in the query chain. 
Currently, rating information is stored inside the AQUA system using our own implementation of rating services. In the near future it is planned to use the SELECT rating server for this purpose. This means that rating information will be globally available, not only within the AQUA system.

\section{Summary}

Integration of information filtering into AQUA has been presented in this paper, which is the first step towards collaboration within AQUA. In the meantime various usability evaluations are carried out to identify how well users are able to understand and apply the query chain paradigm, and what are the possible improvements of the query interface. Usability improvements and experiments with new methods for searching, filtering and collaboration characterize the evolution of AQUA.

\section{References}

1. R. Alton-Scheidl, J. Ekhall, O. van Geloven, L. Kovács, A. Micsik, C. Lueg, R. Messnarz, D. Nichols, J. Palme, T. Tholerus, D. Mason, R. Procter, E.

Stupazzini, M. Vassali, R. Wheeler: "SELECT: Social and Collaborative Filtering of Web Documents and News", 5th ERCIM Workshop on "User Interfaces for All": User-Tailored Information Environments, Dagstuhl, Germany, Nov 28 Dec 11999

2. A. Andreoni, M. B. Baldacci, S. Biagioni, C. Carlesi, D. Castelli, P. Pagano, C. Peters: "Developing a European Technical Reference Library", Proceedings of Research and Advanced Technology for Digital Libraries, ECDL'99, Paris, France, September 1999, Springer LNCS 1696, pp. 343-362

3. AQUA project homepage: http://www.sztaki.hu/sztaki/aszi/dsd/aqua

4. S. Biagioni, J. Borbinha, R. Ferber, P. Hansen, S. Kapidakis, L. Kovács, F. Roos, A. M. Vercoustre: "The ERCIM Technical Reference Digital Library", Second European Conference on Digital Libraries (ECDL'98), Heraklion, Greece, September 1998, Springer LNCS 1513, pp. 905-906

5. L. Kovács, A. Micsik, B. Pataki: "Visualizing queries in a distributed digital library system", Euromicro Workshop on Multimedia and Telecommunications at 26th Euromicro Conference: "Informatics: inventing the future", Maastricht, the Netherlands, September 5-7 2000

6. L. Kovács, A. Micsik: "The Collaborative Web", ERCIM News No. 41, April 2000, Le Chesnay ISSN 0926-4981, pp. 35-36,

URL: http://www.ercim.org/publication/Ercim_News/enw41/kovacs.html

7. L. Kovács, A. Micsik, B. Pataki: "AQUA: Query Visualization for the NCSTRL Digital Library", Proceedings of the 4th ACM Conference on Digital Libraries, August 11-14 1999, Berkeley, California, pp. 230-231

8. L. Kovács: "Discovery of Resources within a Distributed Library System", Communications of the ACM, Vol. 41. No. 4. April 1998

9. B. M. Leiner: "The NCSTRL Approach to Open Architecture for the Confederated Digital Library", D-Lib Magazine, December 1998, ISSN 1082-9873, URL: http://www.dlib.org/dlib/december98/leiner/12leiner.html 